\title{
Laboratory experimentation at the Center for Computer-Based Behavior Studies*
}

\author{
GERALD H. SHURE and ROBERT J. MEEKER \\ University of California, Los Angeles, California 90024
}

\begin{abstract}
The Center for Computer-Based Behavioral Studies (CCBS) is designed around a time-shared computer system to make its informational and technological resources available to behavioral scientists and policy analysts at remote locations (through the ARPA Network and through dial-in phone lines) as well as in our own laboratory. The principal goal of CCBS is to broaden and expand the capabilities and horizons of behavioral research for studying problems of real-world complexity and theoretical relevance. The principal focus is on strengthening laboratory and associated research methodologies through computer-administered multiperson experiments and gaming simulations.
\end{abstract}

The Center for Computer-Based Behavioral Studies (CCBS) is designed around a time-shared computer system to make its informational and technological resources available to behavioral scientists and policy analysts at remote locations (through the ARPA Network and through dial-in phone lines) as well as in our own laboratory.

The principal goal of CCBS is to broaden and expand the capabilities and horizons of behavioral research for studying problems of real-world complexity and theoretical relevance. The principal focus is on strengthening laboratory and associated research methodologies through computer-administered multiperson experiments and gaming simulations. The CCBS approach and developments are described in the following areas: (1) A uniquely designed computer interface that minimizes or eliminates user knowledge of computer procedures or programming languages. (2) A laboratory implementation system to aid in the design, development, checkout, and administration of man-machine prototypes and laboratory studies involving human interaction in complex settings. (3) An integrated, interactive system for data analysis that links all phases of analysis, providing special capabilities in the frequently neglected exploratory phase. (4) A physical laboratory and computer system designed to support all of the preceding activities in an integrated or mutually supportive fashion.

\section{BACKGROUND}

While the Center is barely 4 years old, many of the basic concepts and methods used in its development are a direct outgrowth of experience with two computer-based laboratories at the System Development Corporation.

* An earlier version of this paper was presented at the National Conference on the Use of On-Line Computers in Psychology, St. Louis, Missouri, October 31,1973 . Research reported herein was conducted under Contract $\mathrm{F} 30602-70-\mathrm{C} 0016$ with the Advanced Research Projects Agency, Department of Defense.
Looking at the forerunners of CCBS, it is fair to say that the early computer-based laboratories were built on a belief in the potential of computer technology. The prospect of a large-scale general-purpose laboratory promised the advantages of experiments implemented and managed by a computer, where as many as $24 \mathrm{Ss}$ could be involved in a single experimental session. The computer provided uniformity and precise control of experimental variation as well as exceptional accuracy of record administration. Perhaps most appealing of all, the general-purpose design of the laboratory made the same physical resources accessible to a wide variety of investigative interests. The laboratory has been used for such varied purposes as binary choice experiments, leadership studies, research in computer-aided instruction, man-machine problem-solving studies, bargaining and negotation experiments, organization and management exercises, and simulations of international relations.

In an earlier paper we have described some of the distinctive features and advantages of computer management as they have been used to broaden and improve various aspects of experimentation (Shure \& Meeker, 1970). In the studies cited, we used on-line computer methods for the following:

(1) Systematic assignment of Ss to experimental roles or conditions, based on preexperimental assessment of individual differences among Ss;

(2) Computer-aided presentations of experimental instructions, with tests for comprehension of these instructions to guarantee that $\mathrm{Ss}$ will not enter an experimental situation without a minimal level of understanding;

(3) Preparation of experimental stimuli, based on complex criteria and random sampling of these criteria for presentation;

(4) Varying patterns and proportions of stimulus elements in a nonstationary environment, contingent upon Ss' responses; 
(5) Simultaneous administration of all 24 combinations of a 2 by 2 by 2 by 3 factorial design in a single laboratory session;

(6) Control of one or more sides of a social interaction situation in which relatively simple fixed patterns of response, or complex algorithms, are used to specify the behavior of accomplices in social psychological studies;

(7) Questioning of Ss at various points during an experiment (based on situation-specific events, these questions elicit additional subjective information that helps to clarify his perceptions associated with his behavior);

(8) In-process assistance to Ss performing experimental tasks and to Es monitoring their performance;

(9) Instrumenting, and dynamically varying, complex communication structures;

(10) Exchanging preformatted and open-format messages;

(11) Using dispersed, or remotely located, participants in integrated simulation exercises.

While this list of methods provides a broad and robust environment for a variety of multiperson research formats, it is also important to note that there are a number of kinds of on-line capabilities that are not included in our time-sharing mode of operations. In attempting to make the computer system an optimal environment for the primary research functions of CCBS, the system has explicitly not tried to provide guaranteed response time or to accept as direct inputs physiological or short reaction-time responses. For the present, these capabilities seem better served by special-purpose or smaller dedicated computers.

\section{SOME METHODOLOGICAL IMPLICATIONS}

Of even greater importance than any of the particular features of on-line experimentation was the broadened interpretation of results achieved when the data from these many sources were collected within a single experiment. These multiple-source data were immensely useful, if not essential, for understanding the behavior evoked in a number of laboratory studies (Shure, Meeker, \& Hansford, 1965; Shure, Meeker, Moore, \& Kelley, 1966; Shure \& Meeker, 1969). By providing a large amount of detailed information-pregame ratings, process data, and in-play perceptions and reports by Ss-to supplement gross outcome measures, the computer proved to be an extremely important tool for research on the complex situations represented by our studies. These multiple inquiry data enable the researcher to identify and pinpoint the dynamics of underlying processes, both at the level of the individual (reactions and motivations behind certain actions) and at the interpersonal level (action-reaction effects). Such data proved useful in four ways: (1) to account for the observed effects of experimental treatments, (2) to permit internal analyses, (3) to facilitate cross-study comparisons, and (4) to expose methodological flaws and artifacts likely to be present in laboratory studies, particularly those using human Ss in social interactive settings.

Where apparently similar experiments produce different results, the usual recourse for determining which factor or combination of factors is crucial has been to design successive experimental variations until a consistent answer is obtained. As the laboratory setting becomes more complex, this approach becomes more costly and the results uncertain. An expanded inductive focus in experimentation, using multiple sources of data to augment a narrower hypothesis-testing approach, offers a promising solution to this problem.

\section{THE CCBS USER SYSTEM}

The CCBS system support and services are built around a user group that is more diverse than in most computer centers. In the CCBS system, the primary target user is the experimenter and researcher rather than the programmer. The difference is evidenced mainly in the Center's extensive support of on-line behavioral studies for a user who may have little or no computer or programming knowledge but who wishes to use the Center as a vehicle for designing or conducting experiments, simulation exercises, training, or data analysis. His interests are predominantly substantive, and the laboratory is a means to his ends. By the nature of his enterprise, any direct contact with the laboratory will be more occasional and sporadic than continuous. To provide a capability for users (who cannot be treated as system users in the usual sense, i.e., as applications software developers or as applications software users) requires that they interact with the computer through specially developed Center programs rather than at the operating system level.

This greater diversity of users, and the emphasis on a different class of target user, has required extensive modifications in the CCBS operating system and has led to the development of the CCBS User Package. This package serves as an envelope for all applications programs in the User Package. It provides operating system level services-program transfers, access to system utilities, file maintenance, run-time error returns, etc.-so users need not be concerned with, or distracted by, system level conventions and procedures. In effect, the User Package serves as a constant go-between, allowing one to operate at a functional level above the operating system. The range of capabilities in the CCBS User Package is presented in Fig. 1.

If the potentials of the computer-based experimental vehicle are to be profitably exploited by a wider user community, the first two general sets of capabilities shown in Fig. 1 are needed. These are described in the following sections.

\section{LABORATORY IMPLEMENTATION SYSTEM (LIS)}

Past experience suggests that while investigators may 


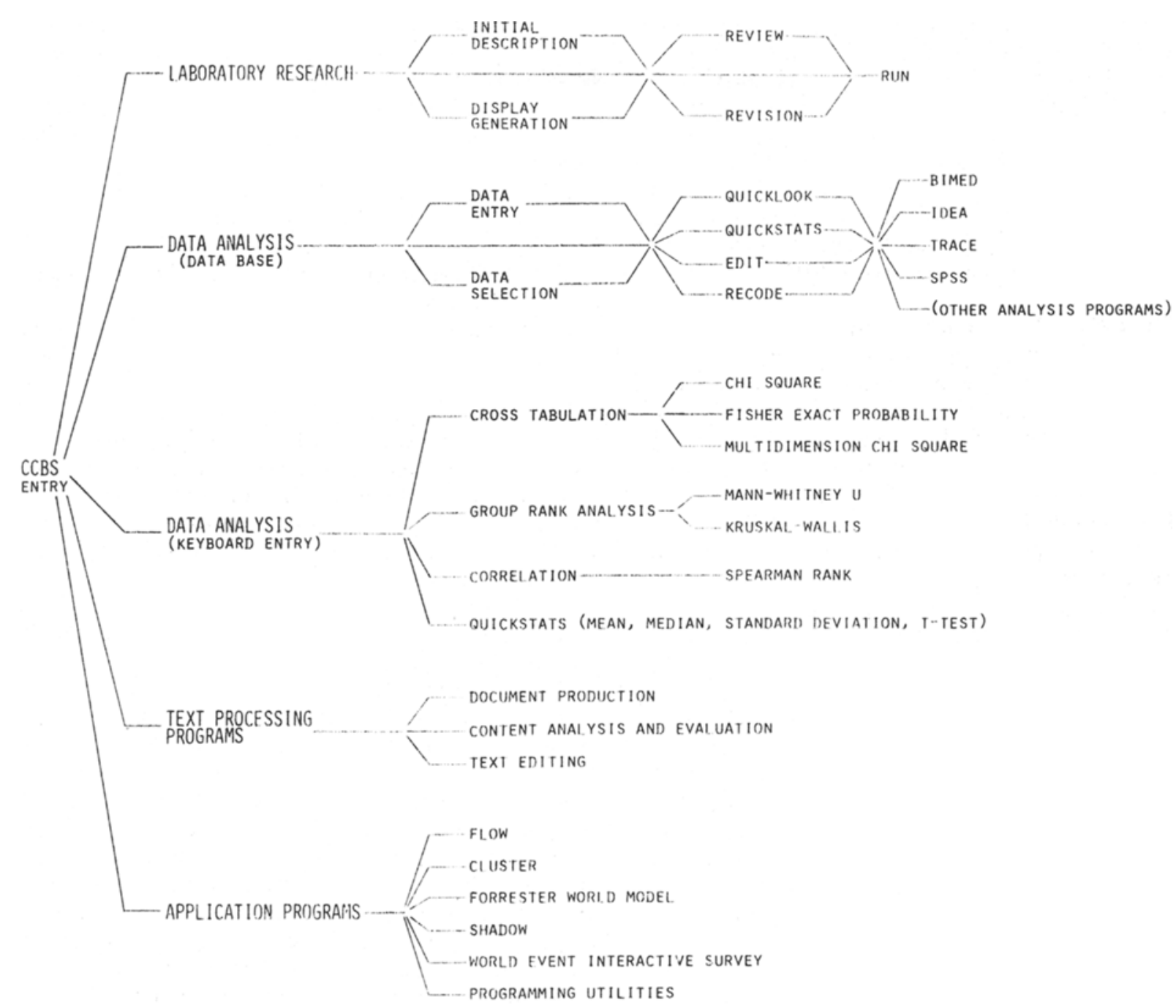

Fig. 1. Overview of CCBS User Package programs.

perceive the potential of the computer-based laboratory, they are fearful of reported obstacles and high development costs. First, the operation itself is not generally well understood. Investigators are not programmers and, for the most part, are unfamiliar with control systems. Second, the lead time before first concrete results emerge seems inordinately long. Most behavioral investigators are accustomed to building their experiments in successive stages, with frequent opportunities for testing and modification. Third, the specification process is unfamiliar. Each experimental vehicle is a program system, requiring specifications to cover all interactive contingencies. The art of producing such specifications is not easily mastered and is not worth learning for the infrequent user. To overcome these barriers, CCBS has developed the Laboratory Implementation System (LIS) as a means of translating investigator requirements into an operational program.

LIS is designed to optimize the investigator's formulation practices by providing him with a general, interactive vehicle; it permits him to operationalize his experimental design logic rapidly and poses formulation problems to him in the most concrete form possible. This approach to the laboratory reduces significantly the design to implementation phase of experimentation development. By providing the experimenter with an opportunity to "breadboard" his experiment, it puts him immediately into an operational posture and permits him, by trial and error, to successively approximate his final research design.

The total LIS system is composed of four programs. These correspond functionally to the natural implementation stages.

\section{LSPEC-Laboratory Specifications Program}

This program presents an interactive questionnaire to the user. Responses to the questionnaire are the basis for generating formal specifications for a "first-cut" version 
of an experiment. The response to specifications is automated. The resulting specifications are sufficient to check out the logic of the experiment; if modifications are required, the system offers an easy transfer to the LISED program (to make necessary changes) and an easy transfer from LISED to LISIM (to reflect the changes made). Iterations permit the user to successively approximate his final research design. Elaboration of the display content is handled through DGP with comparably easy transfers between LISIM and DGP.

\section{LISIM-LIS Implementation Program}

This is the central program in the system. It operates interpretively on table entries that determine routing of information, evaluation of responses, presentation of displays, recording of data, and logic contingencies necessary for implementation of experiments.

\section{LISED-LIS Editing Program}

Since LIS operates interpretively on table entry values, experiments can be modified by changing these values. The LISED program permits the user to edit the tables in a very direct fashion.

\section{DGP-Display Generation Program}

This program is used for preparing displays composed of straight-line graphics and alphanumeric information for use in experiments or for educational purposes. The procedures for preparing displays are conversational. The user language has been designed for the nonprogrammer, and operating instructions are incorporated directly into the program.

A more extended description of the LIS system is reported in Meeker, Shure, and Cooperband (1971).

\section{INTERACTIVE DATA ANALYSIS TOOLS}

The increased capabilities of the computer-based laboratory for multimethod data acquisition leads to data sets which are organized in different ways, hierarchically, sequentially, time-ordered, associated with particular states in a dynamically changing environment, as long sequences of interactions, etc. We have already noted how valuable it may be for the $\mathrm{E}$ to adopt an exploratory stance toward these data. This may involve identification and classification of patterns of response, iteratively refining of operational definitions, varying the data subsets used in the course of the analysis to check hypotheses about relationships within these particular subsets, linking data units that exist at different levels, and readjusting his analytic framework according to suggestions contained in earlier analyses.

To forego these opportunities for exploratory analysis is to lose much of the potential value of the computer's expanded data collection capabilities. Furthermore, it assumes far too much about the present state of knowledge of social processes in laboratory studies and about the investigator's consequent ability to determine in advance the most appropriate process indices.
To provide the more powerful capabilities for the range of general-purpose capabilities noted above, and to do so in an interactive mode that minimizes the burden of data administration and programming knowledge, three systems were developed and incorporated in the User Package in a functionally integrated fashion: PREP, TRACE III, and IDEA. These are discussed next.

\section{PREP}

The PREP system functions as the CCBS interface to generally available statistical programs that are typically run in a batch processing mode. PREP is a system of computer programs providing a common data base interface to data analysis programs and packages. The PREP system provides a high degree of user support that minimizes the need for user knowledge of programming conventions and operating system functions. The user need enter and edit his data only one time to use any of the data analysis programs that interface with the PREP system. The necessary, but usually troublesome and error-laden, process of data preparation (entry, verification, and prestatistical manipulation) is concentrated and standardized for all application programs supported by the PREP system.

The distinguishing feature of PREP is that it concentrates analysis preparation functions and yet remains an open-ended system in terms of the analysis programs that it supports. This contrasts on the one hand with "integrated" systems, like SPSS (Nie, Bent, \& Hull, 1970) and DATATEXT (Armor \& Couch, 1972), which provide data preparation capability but are essentially limited to the analysis programs included in the "integration package"-i.e., it is very difficult to add new statistical programs to these packages. Furthermore, PREP provides considerably more user-oriented and interactive support in analysis preparation than either of these "integrated" packages, and does so in a manner that makes it possible to apply these capabilities to an easily expandable set of data analysis and statistical programs. In this respect PREP is best viewed as an "interfacing system" rather than as an integrated package.

PREP contrasts, on the other hand, with a large set of "stand alone" analysis programs in statistical library packages (e.g., BMD, Dixon, 1967) in which each program provides a unique set of data preprocessing and preparation functions required for its own use. As a consequence, these capabilities are typically limited in scope and not standardized from one program to the next. For the user, this means (1) he must learn to use each program on its own terms, and (2) he cannot transfer the results of the data preparation from one program to another. In summary, PREP (1) concentrates this learning process (one set of procedures for all applications), (2) reduces the need for learning, by giving the user considerably greater assistance in the analysis preparation tasks, and (3) makes the results available to all interfacing analysis programs.

The PREP system incorporates four major processing 
functions-input, verification check, editing, and interface. The input function accepts data from the conventional sorts of machine-readable storage mediums, producing a transposed data structure for the greater efficiency of selected data retrieval. The verification check offers a variety of "quick-look" data summaries as a means of verifying the accuracy of the original and stored data. The editing function provides a number of procedures for error correction, data recoding, and data transformation. The interface function is, of course, different for each of the programs being interfaced to the PREP system (and each must be separately produced), but since they all relate to a standard data structure, there is enough commonality to make it a clean and practical programming task to bring most standard existing or new data analysis programs into the system.

The PREP system, implemented in standard FORTRAN IV, has a high degree of transferability to other computing installations. In this regard, PREP requires no special operating system support, and if desired, it permits the analysis programs it supports to be used in the conventional manner, thus making the same analysis programs accessible to both experienced and naive users.

\section{IDEA}

A computer program for Inductive Data Exploration and Analysis of a multivariate data base was designed for discovering and summarizing potentially interesting data models in the form of restricted tree structures. These graphically represent the rule used to partition a set of observations into exhaustive, mutually exclusive regions. IDEA was developed for on-line interaction in a time-shared computer environment through hard copy terminals or cathode ray tube displays to permit the investigator to collaborate with a library of programmed heuristics in the process of uncovering and representing the structure in his data (Shure, Press, \& Rogers, 1968; Press, Rogers, \& Shure, 1969).

Classical induction algorithms, such as regression analysis, discriminant analysis, and cluster analysis, are often used by an investigator to help him discover, understand, summarize, and represent various relationships that exist among many measurements and observations. Whenever the totality of such relationships becomes very complex, containing nonlinearity and interaction, the usefulness of these approaches is limited. Furthermore, in many cases, the emergent structure is not obvious to the researcher, and he is not solely interested in testing an a priori hypothesis. If, under these conditions, his experience, knowledge of the field from which the data come, intuition, and pattern recognition skills could be utilized, he would be able to improve the results of classical methods or perhaps succeed where these techniques fail (Press, 1967).

To supplement traditional multivariate techniques, a few investigators have used decision trees to represent data with the above mentioned characteristics (Hunt,
Marin, \& Stone, 1966; Sonquist, Baker, \& Morgan, 1971). Rather than summarizing structure with a single complex relationship, such programs divide the data into sequential subsets in which the criterion is simply predicted by a unique model for each unique region of the data space. The most concise form of prediction function is the $i^{\text {th }}$ subset is a constant plus random noise: $y_{i}=k_{i}+E$. Computer routines search for homogeneous criterion subsets (e.g., all blue-eyed males have high IQs). One of the important results of this general approach is that the sequential partitioning uncovers relationships between a dependent and a predictor variable that are obscured by a third variable (e.g., if blue-eyed males have high IQs and blue-eyed females have low IQs, a mixed data set of equal numbers of males and females will not yield a relationship between eye color and IQ unless it is partitioned into male and female subsets).

Since the number of decision trees that could result from a practical data base is large, the routines that search for structure are of necessity heuristic. They seek out a subset of candidate variables for partitions at each node. These "promising" partitions are then evaluated by means of heuristically chosen criteria according to how successfully a partition can account for the variation in the dependent variable. The program recommends which independent variable to use at each node and how to group the values for that variable; the user retains the option to choose some other variable or a different set of partitions for the recommended variable. By providing in-process feedback and the opportunity to introduce his own criteria in the induction process, IDEA expands the utility of the tree structure concept and helps overcome some of the restrictions in similar pure machine induction programs.

The process used at the first step in the analysis, when the entire data base or a random sample thereof is considered, is the same as that for subsets at lower points in the tree. Termination decisions are based on arbitrary thresholds for desired quality of fit, sample size, and improvement in quality of fit.

\section{TRACE}

TRACE is an extremely general and powerful data analysis, management, and retrieval system. Because of a flexible data management substratum to a powerful command language, TRACE can be viewed as a convenient implicit programming system. Its very generality, however, results in higher overhead and requires a more skilled user than do the other data analysis programs in PREP. For this reason, TRACE is viewed as a special tool for attacking exceptional problems not easily handled by the other CCBS capabilities.

One set of such problems is related to large and/or hierarchically related data structures, particularly when the user needs to restructure all or parts of such data structures or deal with them at various levels of data aggregation. A different, but intersecting, set of 
problems centers on the need to perform quite involved operations on data, and utilizing arbitrarily complex algebraic and nonalgebraic manipulations.

The set of capabilities in TRACE is so large, and operations can be combined in so many ways, that PREP-type user protocols would limit the range of services conveniently available to the user. Once connected to TRACE, the user is expected to control its operation by issuing commands in the TRACE language and respond ing to requests for information.

TRACE, similar to the CCBS User System, appears as a single, monolithic program, although it is composed of a number of components, three of which are particularly important. The first is a data base input package. This function either accepts a PREP data base or raw data sets and generates a TRACE archive. Data in a TRACE archive are represented in transposed form, having all values encoded, binary packed, and associated with code books and other summary and descriptive information.

The second component, and the one with which the user interacts the most, is the TRACE compiler. This program is a syntax-directed compiler that parses each command from the user as it is entered, line by line. Syntactic and semantic errors are identified and fed back to the user. A dimensional analysis is performed on the data structures implicit in the command. When a well-formed error-free command has been parsed, the compiler generates a "TRACE machine language program" representing that command in a form that can be followed by the third major component, the TRACE interpreter.

The interpreter actually does most of the work in the TRACE system. It retrieves values of those variables required to satisfy the user's command and arranges them in a multidimensional array whose structure is implied by the command. Then the data manipulations are applied iteratively to all cells of this array and the results either printed out or returned to the data base as new variables. An important feature of the TRACE system is that the structure of the data array created for any command is determined not by any fixed structural relationships in the data archive but by the operations that are to be performed and by the values of the variables operated on. In a sense, the working structures within TRACE are semantically determined, not syntactically fixed. No assumptions are made either about the structural relationships among the variables in an archive or about the hierarchical relationship of different data sets, except that associated values of a set of variables are treated as representing a single observation. Because of this, the user is free to view his data from whatever perspective he pleases and can treat his data at whatever level of aggregation is suitable; it also makes it possible to return new computed variables to the data base or add new kinds of observations to the data base. Once a derived variable is returned to the data base, it becomes functionally equivalent to variables, input from raw data sets.

The TRACE command language uses a compact, powerful notation that is easy to learn because of its similarity to conventional algebraic, logical, and statistical notation. Although the language is rich in terms of the number of different operators available and the ways they can be combined, it is simple in that there are very few syntactically different forms. Almost all but routine data base editing operations fall into a single general form in which the user first declares the dimensions of a data space within which a set of manipulations is to be performed and then supplies the manipulations. In a sense, the user writes special-purpose data manipulations programs in a TRACE programming language, but one where TRACE assumes responsibility for most of the error-prone aspects of programming. What is left is a language that is very close to the data and the operations, avoiding almost all of the concerns for data structuring and process control-and their idiosyncratic notation-that characterize typical programming languages (Cooperband, 1970; Cooperband, Moore, Meeker, \& Shure, 1971).

\section{DESCRIPTION OF LABORATORY}

\section{Laboratory Facilities}

The CCBS research facility, completed in 1972, is located in the Psychology Department on the UCLA campus and provides space for the computer, laboratory cubicles, briefing and control rooms, and staff offices. While CCBS is often referred to as a "laboratory," it is not intended to support only experimental research. It is designed instead to support a broad range of research and educational activities-behavioral simulation, symbolic simulation, data management and retrieval, computer-aided theory development, and computer programming-as well as an interface for these various activities. There is explicit provision for developmental as well as research and applied phases. Hardware and software configurations have been designed for both experiment preparation and support and for the data-gathering phases of research.

The design principle of the Center makes space maximally flexible: almost all areas serve a number of functions, and almost all equipment is portable to permit reconfiguration.

The laboratory area-about one-fourth of the 7,000 -sq-ft facility-is built around $6 \times 8 \mathrm{ft}$ modules that are connected with the general control system by a number of types of communication lines. There are 24 functionally identical modules, accessible from a perimeter or center corridor. Each module is serviced with line power, separately controlled air conditioning, video lines, low- and high-level audio lines, digital communication lines, digital signaling lines, and telephone lines. All services to a module terminate either in the ceiling or in a corridor wall, and, except where a module abuts on a corridor, all partitions are readily demountable. This design permits overnight reconfiguration of the space-from $246 \times 8 \mathrm{ft}$ rooms to 


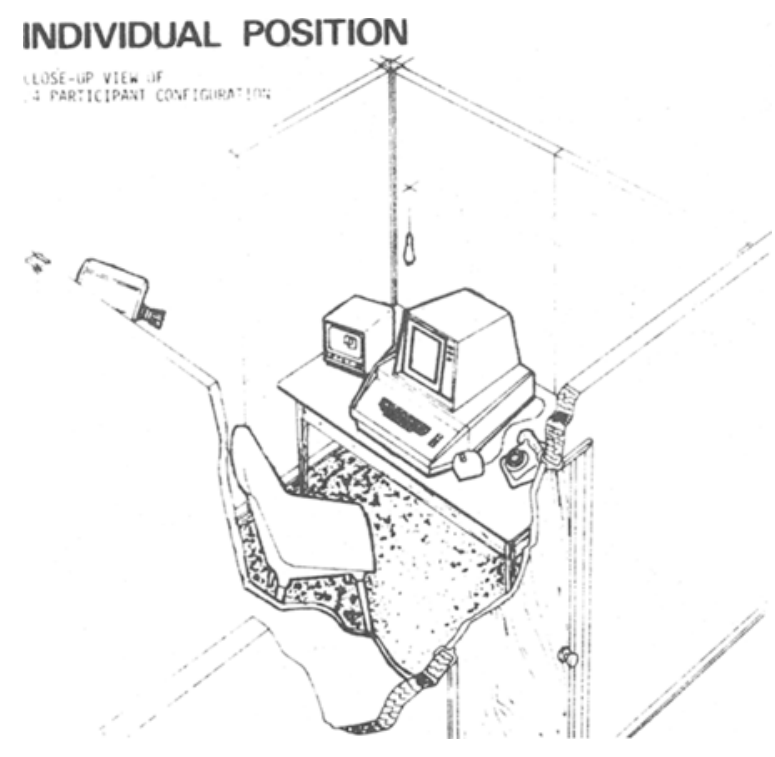

Fig. 2. Experimental laboratory cubicle in CCBS.

$236 \times 16 \mathrm{ft}$ rooms, or into any intermediate configuration.

Any of the laboratory modules can be used as an "experimenter" room. If all laboratory modules are needed for participants, staff office space, the briefing room, and the master control room can also be used. The movable module walls can take one-way glass inserts to provide full view observation of participant areas and have excellent sound-attenuating properties.

\section{Computer Hardware}

For CCBS, the operating environment is determined primarily by: (1) the need for high-speed communications (including graphics capability) for both local and remote users; $(2)$ the need to process large files of symbolic or coded information with a correspondingly limited capability to perform extensive algebraic computations; and (3) the need to obtain computer control over experiments with large numbers of on-line participants. The first two requirements especially have led to several hardware innovations and modifications of the standard operating system supplied with the computer.

Central to the laboratory is a dual processor computer-a PDP-10/PDP-15--with about one-quarter of a million words of high-speed core storage, five disk-pack drives with 5.2 million words storage per pack, and the usual complement of peripherals including two swapping drums, three tape drives, card punch and reader, and a printer plotter. The entire system is time-shared through the PDP-10 operating system. The PDP-15 serves primarily as a communications concentrator to relieve the central processor of the unusually high demands that would be generated by the simultaneous use of the 24 high-speed terminals operating at their maximum rates (4,000 characters per display screen, presented in approximately $6 \mathrm{sec}$ ).

CCBS uses three types of communication terminals--an Ann Arbor terminal with display densities of 24 lines of 80 alphanumeric characters, the Computek Model 400/15 with graphic capabilities and display densities of 55 lines of 80 characters, and the hard-copy Texas Instrument Model 75. Each terminal operates at two or more transmission rates, depending on whether communication lines are hard wired, over the ARPA Network, or connected through acoustic coupling. All terminals use standard keyboard entry. Choice of terminal type is a function of experimental demand, depending upon whether graphics are required, the amount and rate of material desired for presentation on a single display, or whether a direct hard-copy output is desired.

CCBS interfaces with the ARPA Network via the PDP-15. As a result, the CCBS dial-in user to a remote host computer is achieved without any involvement of the PDP-10. The facilities of CCBS are now available nationwide via the ARPA Network as well as to the dial-in user. Additionally, the resources of other network centers are now accessible to CCBS.

\section{CCBS FACILITY FLOOR PLAN}

Fig. 3. CCBS facility floor plan.

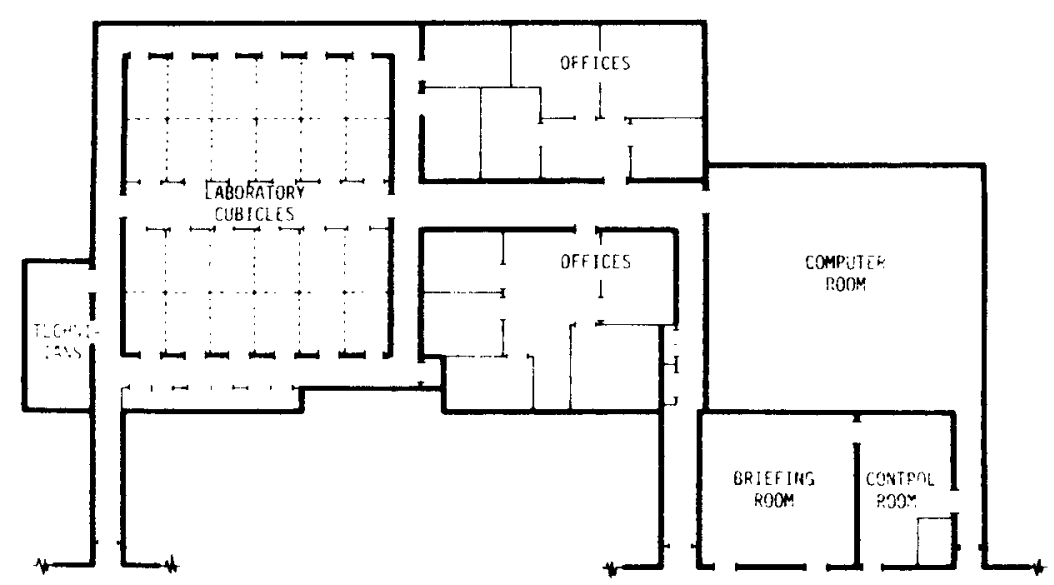




\title{
REFERENCES
}

Armor, D. J. \& Couch, A. S. Data-text primer: An introduction to computerized social data analysis. New York: Frse Press. London: Collier-Macmillan, 1972.

Cooperband, A. S. Basic TRACE III. CCBS Document TM-6, July 31,1970 .

Cooperband, A. S., Moore, W. H., Jr., Meeker, R. J., \& Shure, G. H. TRACE-III: An implicit programming system for inductive data analysis. Proceedings of the 1971 Annual Conference of the Association for Computing Machinery, 1971, 133-138.

Dixon, W. J. (Ed.) BMD biomedical computer programs. Berkeley and Los Angeles: University of California Press, 1967.

Hunt, E., Marin, J., \& Stone, P. Experiments in induction. New York: Academic Press, 1966.

Meeker, R. J., Shure, G. H., \& Cooperband, A. S. An implementation system for designing computer-based experiments. Proceedings of the 1971 Annual Conference of the Association for Computing Machinery, 1971, 169-178.

Nie, N. H., Bent, D. H., \& Hull, C. H. Statistical package for the social sciences. New York: McGraw-Hill, 1970.

Press, L. I, IDEA: A technique for inductive data exploration and analysis. Unpublished doctoral dissertation, University of
California, Los Angeles, 1967.

Press, L. I., Rogers, M. S., \& Shure, G. H. An interactive technique for the analysis of multivariate data. Behavioral Science, 1969, 14, 364-370.

Shure, G. H., \& Meeker, R. J. Bargaining processes in experimental territorial conflict situations. Peace Research Society (International), 1969, 11, 109-122.

Shure, G, H. \& Meeker, R. J. A computer-based experimental laboratory. American Psychologist, 1970, 25, 962-969.

Shure, G. H., Meeker, R. J., \& Hansford, E. A. The effectiveness of pacifist strategies in bargaining games. Journal of Conflict Resolution, 1965, 9, 106-117.

Shure, G. H., Meeker, R. J., Moore, W. H., Jr., \& Kelley, H. H. Computer studies in bargaining behavior: The role of threat in bargaining. SDC Document SP-2196, 1966, System Development Corporation, Santa Monica, Calif.

Shure, G. H., Press, L. I., \& Rogers, M. S. Man-computer derivations of tree structures from multivariate data. Proceedings of the $76 \mathrm{th}$ Annual Convention of the American Psychological Association, 1968, 3, 215-216.

Sonquist, J. A., Baker, L. B., \& Morgan, J. N. Searching for structure. Ann Arbor, Mich: Braun-Brumfield, 1971.

\section{Project IMPRESS, several perspectives: Interactive computing and data analysis*}

\author{
PAUL VELLEMAN \\ Department of Statistics, Princeton University, Princeton, New Jersey 08540
}

\begin{abstract}
Project IMPRESS is an interactive social science data analysis system used extensively at Dartmouth College and throughout the DTSS network. The programming techniques used to make it an unobtrusive time-sharing job and the user interface design considerations used to make it a system easy for both students and experienced researchers to run are described and their pedagogical and research values discussed.
\end{abstract}

Time-sharing can provide a computing environment ideal for analyzing data and for teaching data analysis. Project IMPRESS is a large interactive data analysis system designed to facilitate both of these activities. In the 5 years since development began, it has become an important part of social science teaching and research at Dartmouth College and has been used widely by the schools and research organizations connected to the Dartmouth Time-Sharing System. ${ }^{1}$

IMPRESS is an acronym for Interdisciplinary Machine Processing for Research and Education in the Social Sciences. It consists primarily of a collection of interrelated programs for data analysis, an on-line archive of over 60 cleaned and processed data sets, and programs for retrieving and reformatting variables selected from these data sets. Provisions are also made for users to clean and process their own data and for communication with user-written data analysis programs.

Perhaps the greatest achievement of Project IMPRESS is the demonstration that such a system can exist: (a) Computer-naive undergraduates use it to learn both

* This work is partially supported by an Atomic Energy Commission contract, No. At-11-1-2310, awarded to the Department of Statistics, Princeton University. social science and data analysis. (Student use accounted for $69 \%$ of the more than 20,000 recorded runs in the past year.) (b) Useful data analysis can be done in little time and at reasonable cost. (The average run in this period took $11 \mathrm{~min}$ and cost $\$ 1.81$ at educational rates.) (c) IMPRESS coexists with other time-sharing users without overtaxing the system. (The Dartmouth Time-Sharing System, DTSS, regularly handles over 140 users simultaneously. ${ }^{2}$ )

These achievements required a careful balancing of conflicting pressures in the design of the user interface and the development of sophisticated data manipulation techniques.

\section{COMPUTING ENVIRONMENT}

The early computers liberated social scientists from the drudgery of mechanical calculators and counting sorters and made possible many techniques previously unthinkable. The interactive computer has the ability to free us from the black box, computer code syntax, one-shot-at-a-time style of working, and to recover one of the advantages of the hand calculator: hands-on data manipulation. It has also permitted the development of 